\title{
26392 - PLACENTA PREVIA : A 3 YEAR RETROSPECTIVE STUDY
}

\section{Arivan Govindarajan FRCA, Robina Akhtar, FRCA; Karthik Sabapathi, FRCA; University Hospital Of North Staffordshire, Stoke-on-Trent, STAFFORDSHIRE, UNITED KINGDOM}

Introduction: Women with placenta previa are at high risk from morbidity and mortality from major obstetric haemorrhage. Over the recent years, intraoperative cell salvage has been increasingly recommended in obstetrics. We reviewed the anaesthetic management of caesarean section for placenta previa in our maternity unit. Additionally, we wished to identify risk factors for major blood loss in this group where setting up a cell saver in anticipation would be cost effective.

Methods: We did a retrospective study from September 2002 to August 2005, only including those patients with placenta previa who required a caesarean delivery. Patients were identified from the theatre record books. Details collected were: Maternal age, parity, weeks of gestation, urgency of caesarean section, type of anaesthetic, grade of anaesthetist, grade of placenta previa, previous caesarean delivery, estimated blood loss, blood transfusion in the intraoperative and immediate post-operative period, pre and postoperative haemoglobin.

Results: Of the 15,033 deliveries, there were 70 patients with placenta previa one patient with placenta accreta. Nearly half of the patients had an emergency caesarean section $34 / 70(48.6 \%)$ and the rest had an elective caesarean section 36/70 (51.4\%). The senior most anaesthetist present was a consultant in 42/70 (60\%) patients and either a trainee or staff grade anaesthetist in 28/70 (40\% patients). History of at least one previous caesarean section was present in $24 / 70$ (34\%) patients. Regional anaesthesia (RA) was given to 47 $/ 70(67 \%)$ patients and general anaesthesia (GA) was given to $23 / 70(33 \%)$ patients. Blood transfusion rate was $10 \%$ (7/70 patients). Patients who had a GA not only had a higher mean intraoperative blood loss compared to those who had a RA.(GA $1147 \mathrm{mls}$ Vs RA $773 \mathrm{mls}$ ) but also were almost 3 times more likely to be transfused than those patients who had RA. (GA 4/23 (17.3\%) transfused compared to RA 3/47 (6.3\%) patients).Two patients with multiple previous caesarean sections had massive blood loss and needed emergency caesarean hysterectomies. Out of the 8 patients who had a blood loss of more than $1000 \mathrm{mls}, 5$ patients $(62 \%)$ had a grade 4 placenta previa. Conclusion: Our study reaffirms that regional anaesthesia is a safe anaesthetic technique for caesarean delivery for majority of placenta previa. Our regional anaesthesia rate $(67 \%)$ was comparable with previous studies(1,2).Patients given GA tended to bleed more and required frequent transfusions. We found an association between multiplicity of previous caesarean sections, having a higher grade of placenta previa, receiving a GA and tendency for major blood loss, these patients may be candidates for intraoperative cell salvage. Further studies are needed in this high risk group to prove the strength of their association.

References:

(1)BJA 2000; 84(6): 725-730.

(2)AJOG 1999; 180:1432-1437. 